\title{
(2) OPEN ACCESS \\ Roles of LINC01473 and CD74 in osteoblasts in multiple myeloma bone disease
}

\author{
Fengping Peng (ㄷ) , Siyang Yan, Hui Liu, Zhaoyun Liu 두 , Fengjuan Jiang, \\ Panpan Cao, Rong Fu
}

Additional supplemental material is published online only. To view, please visit the journal online (http://dx. doi.org/10.1136/jim-2021002192).

Department of Hematology, Tianjin Medical University General Hospital, Tianjin, China

\section{Correspondence to}

Dr Rong Fu, Tianjin Medical University General Hospital, Tianjin, Tianjin, China; furong8369@tmu.edu.cn

Accepted 6 January 2022

Check for updates

(C) American Federation for Medical Research 2022. Re-use permitted under CC BY-NC. No commercial re-use. Published by BMJ.

To cite: Peng F, Yan S, Liu H, et al. J Investig Med Epub ahead of print: [please include Day Month Year]. doi:10.1136/jim2021-002192

\section{ABSTRACT}

The suppression of osteoblast (OB) activity is partially responsible for multiple myeloma (MM) bone disease. Long non-coding RNAs (IncRNAs) play a vital role in bone formation and resorption. However, their functions in OBs from patients with MM have rarely been reported. Through highthroughput sequencing of $\mathrm{OBs}$ from patients with MM and healthy controls, we identified several IncRNAs and messenger RNAs (mRNAs) with different expression profile and validated them using quantitative real-time PCR. In total, 22 upregulated and 21 downregulated IncRNAs were found in OBs from patients with MM. Moreover, 18 upregulated protein-coding mRNAs were identified. The expression levels of LINC01473 and its associated co-expression mRNA, CD74, were higher in patients with $\mathrm{MM}$ than in healthy controls ( $p=0.047$ and $p=0.016$, respectively). LINC01473 expression demonstrated a negative correlation with serum interleukin-2 and tumor necrosis factor $\alpha$ levels, whereas the expression of mRNA CD74 was positively associated with serum lactic dehydrogenase in patients with MM. Aberrant expression of IncRNAs and mRNAs was observed in OBs from patients with MM. This study identifies new promising targets for further research on imbalanced bone formation and resorption and MM immune escape.

\section{INTRODUCTION}

Multiple myeloma (MM) is a hematological malignancy characterized by the proliferation of abnormal plasma cells in the bone marrow, resulting in the production of monoclonal immunoglobulin, substantial immunosuppression, and end-organ damage; the key clinical and laboratory features are anemia, bone lesions, renal failure, and hypercalcemia (CRAB symptoms). ${ }^{1}$ Approximately $80 \%$ of newly diagnosed patients with MM develop bone disease, ${ }^{2}$ such as substantial bone pain and pathological fractures, and the need for therapeutic intervention. Overactive osteoclast and reduced osteoblast (OB) activities are responsible for pathological bone remodeling. ${ }^{3} \mathrm{MM}$ cells and osteocytes restrained $\mathrm{OB}$ activity by expressing winglesstype antagonists such as sclerostin, Dickkopf-1, and soluble frizzled-related proteins. ${ }^{45}$ Furthermore, activin-A, semaphorin-4D, and cytokines

\section{Significance of this study}

What is already known about this subject?

- The suppression of osteoblast activity is partially responsible for multiple myeloma bone disease.

- Long non-coding RNAs play a vital role in bone formation and resorption.

What are the new findings?

- We demonstrated that the expression levels of LINC01473 and its associated co-expression messenger RNA, CD74, were elevated in patients with multiple myeloma using high-throughput sequencing for osteoblasts.

How might these results change the focus of research or clinical practice?

- New promising targets to prevent and treat multiple myeloma bone disease were identified, which will advance research on imbalanced bone resorption and formation and multiple myeloma immune escape.

(secreted by bone marrow stromal cells, MM cells, osteoclasts, and immune cells) suppressed OB production, differentiation, and function. ${ }^{6-8}$

Long non-coding RNAs (lncRNAs) play an important role in the development and maintenance of cellular phenotypes, including chondrocytes and osteocytes. ${ }^{9}$ Located in the nucleus or cytoplasm, lncRNAs are non-coding transcripts over 200 nucleotides in length. ${ }^{10}$ Recently, lncRNAs have gained popularity for further studies on the pathogenesis of tumors diseases rather than on the structural remnants. It has been demonstrated that many aberrant IncRNAs are expressed during MM progression. These aberrantly expressed lncRNAs have many functions, including participating in cell proliferation and apoptosis, ${ }^{11}$ interacting with microRNA, protein-coding genes, and myeloma progression. ${ }^{12-14}$ Increasing evidence has shown that IncRNAs participate in osteogenesis. Researchers have identified several lncRNAs (such as HOTAIR, H19, DANCR, SIRT1, and MEG3) that are involved in osteogenesis, and these lncRNAs can regulate osteogenic markers or key regulatory factors in the pathways of 


\begin{tabular}{lc}
\hline Table 1 & \multicolumn{1}{c}{ Clinical data of patients with MM } \\
\hline Characteristics & Patients \\
\hline Number & 23 \\
Gender & \\
\hline Male & $14(61 \%)$ \\
\hline Female & $9(39 \%)$ \\
\hline Age (year) & $67(45-76)$ \\
\hline Types of MM & lgG (12), IgM (1), IgA (3), light- \\
& chain (6), non-secretory (1) \\
\hline Stage of pretreatment (ISS) & \\
\hline I & $8(35 \%)$ \\
\hline II & $9(39 \%)$ \\
\hline III & $6(26 \%)$ \\
\hline Bone scanning of pretreatment & \\
\hline Group A & $3(13 \%)$ \\
\hline Group B & $20(87 \%)$ \\
\hline Parameters of pretreatment & $13(57 \%)$ \\
\hline Creatinine clearance $>30 \mathrm{~mL} / \mathrm{min}$ & $10(43 \%)$ \\
\hline Creatinine clearance $\leq 30 \mathrm{~mL} / \mathrm{min}$ & $9(39 \%)$ \\
\hline Hemoglobin $\geq 100 \mathrm{~g} / \mathrm{L}$ & $14(61 \%)$ \\
\hline Hemoglobin $<100 \mathrm{~g} / \mathrm{L}$ & $14(61 \%)$ \\
\hline Calcium $>2.75 \mathrm{mmol} / \mathrm{L}$ & $9(39 \%)$ \\
\hline Calcium $\leq 2.75 \mathrm{mmol} / \mathrm{L}$ & \\
\hline
\end{tabular}

ISS, International Staging System; MM, multiple myeloma.

osteogenic differentiation. ${ }^{15-21}$ In addition, lncRNA can also be used as a biomarker to regulate some of the key regulatory factors in osteogenesis. ${ }^{22}$

The effects of altered lncRNA expression in OB proliferation and differentiation during the pathogenesis of $\mathrm{MM}$ bone disease have not yet been reported, and hence, further research is required. In this study, we used lncRNA and messenger RNA (mRNA) high-throughput sequencing for OBs from the bone marrow of patients with MM.

\section{MATERIALS AND METHODS} Study participants

From September 2019 to August 2020, 23 newly diagnosed patients with MM hospitalized in the Department of Hematology, Tianjin Medical University General Hospital, were enrolled in this study, according to the National Comprehensive Cancer Network guidelines for $\mathrm{MM}^{23}$ Based on the whole-body CT or FDG PET/CT pretreatment data, patients were divided into two groups: group A, without osteolytic damages or osteoporosis alone; and group B, with osteolytic damages and/or a pathological fracture. Patient information is shown in table 1. Fifteen volunteers (median age, 52 years; range, 4474 years) were recruited as healthy controls.

\section{OB cell culture}

We collected bone marrow extracts from patients with MM and healthy controls. The bone marrow-derived mononuclear cells (BMMNCs) were separated and cultured in Dulbecco's Modified Eagle Medium/F12 medium (Gibco, Australia), $15 \%$ fetal bovine serum (Gibco), $100 \mathrm{U} / \mathrm{mL}$ penicillin and streptomycin, $0.01 \mathrm{~mol} / \mathrm{L} \beta$-sodium glycerophosphate, $10^{-7} \mathrm{mmol} / \mathrm{L}$ dexamethasone, and $0.05 \mathrm{~g} / \mathrm{L}$ vitamin $\mathrm{C}$, at $37^{\circ} \mathrm{C}$ and $5 \% \mathrm{CO}_{2} \cdot{ }^{24}$ The OBs were collected after two subcultures. Osteogenic differentiation was confirmed by the detection of alkaline phosphatase activity and Von Kossa staining as previously described. ${ }^{2425}$

\section{IncRNA and mRNA high-throughput sequencing}

RNAs from five paired OB samples from MM and healthy control were separately extracted using TRIzol reagent (Invitrogen) and frozen at $-80^{\circ} \mathrm{C}$ until analysis. The quantity and quality of RNA were tested using a NanoDrop 2000 spectrophotometer. RNA integrity was assessed using an Agilent 2100 bioanalyzer. Library construction and deep sequencing was performed at Shanghai Genechem using the Illumina PE150 platform according to the manufacturer's instructions. The profiles of $\operatorname{lncRNAs}$ and mRNAs in OBs from $\mathrm{MM}$ and healthy controls were analyzed using the $\mathrm{R}$ software package DESeq2. An adjusted $\mathrm{p}$ value $<0.05$ and $\log 2$ fold change absolute value $>2$ were used to indicate differential expression. The datasets generated for this study can be found in the Sequence Read Archive public repository: https://www.ncbi.nlm.nih.gov/sra/PRJNA760661. ${ }^{26}$

\section{Gene Ontology and Kyoto Encyclopedia of Genes and Genomes pathway analyses}

To explore the mechanisms of $\mathrm{OB}$ inhibition in MM, we annotated all the differentially expressed lncRNAs and mRNAs using Gene Ontology (GO) and Kyoto Encyclopedia of Genes and Genomes (KEGG) pathway analyses. GO enrichment analysis categorized gene function into biological processes (BP), cellular components (CC), and molecular functions (MF). KEGG pathway analysis identified high levels of function and interactions between differentially expressed genes (DEGs) to understand biochemical reactions, signaling pathways, metabolic pathways, and biological processes.

\section{Quantitative real-time PCR}

OB RNA from 18 patients with MM and 10 healthy controls was isolated and synthesized. The primer sequences of candidate genes are presented in online supplemental table S1. The primers were purchased from Sangon Biotech (Shanghai, China), and quantitative real-time PCR was performed using the Bio-Rad iQ 5 Real-time system (BioRad, USA). The amplification protocol for lncRNAs and mRNAs was: $95^{\circ} \mathrm{C}(30 \mathrm{~s}), 95^{\circ} \mathrm{C}(5 \mathrm{~s})$, and extension $(30 \mathrm{~s})$ at different temperatures for 45 cycles (online supplemental table S1). Thereafter, we calculated the relative expression levels of lncRNAs and mRNAs using $2^{-\Delta \Delta \mathrm{Ct}}$ method with GAPDH as the reference gene.

\section{Statistical analysis}

Analysis of the differences between both control and test groups was performed using the Student's t-test with the GraphPad Prism V.5.0 software. Statistical significance was set at $\mathrm{p}<0.05$.

\section{RESULTS \\ IncRNA and mRNA expression in OBs of patients with $M M$ and healthy controls}

lncRNA and mRNA high-throughput sequencing was performed to identify abnormal candidates by using five age-matched and sex-matched pairs from patients with 
(A)

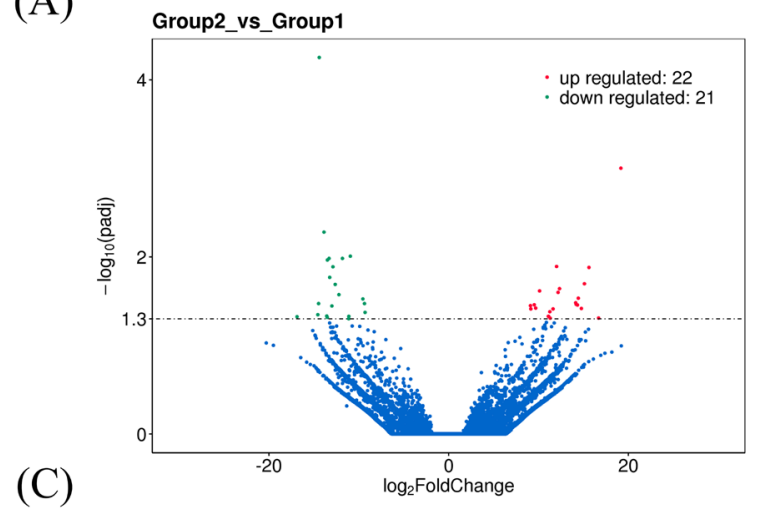

(C)

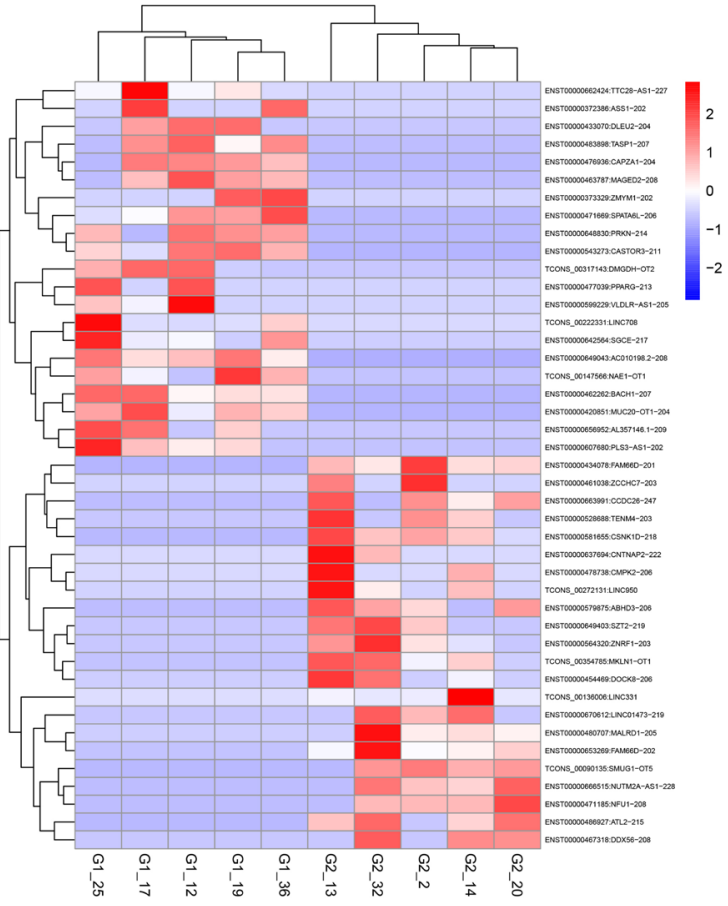

(B)

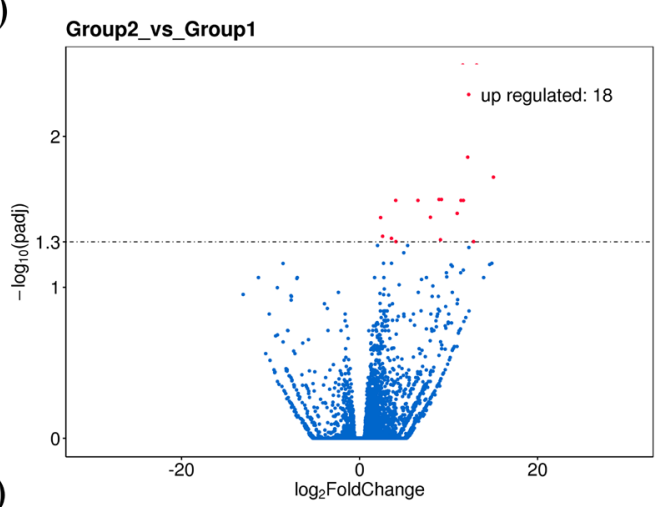

(D)

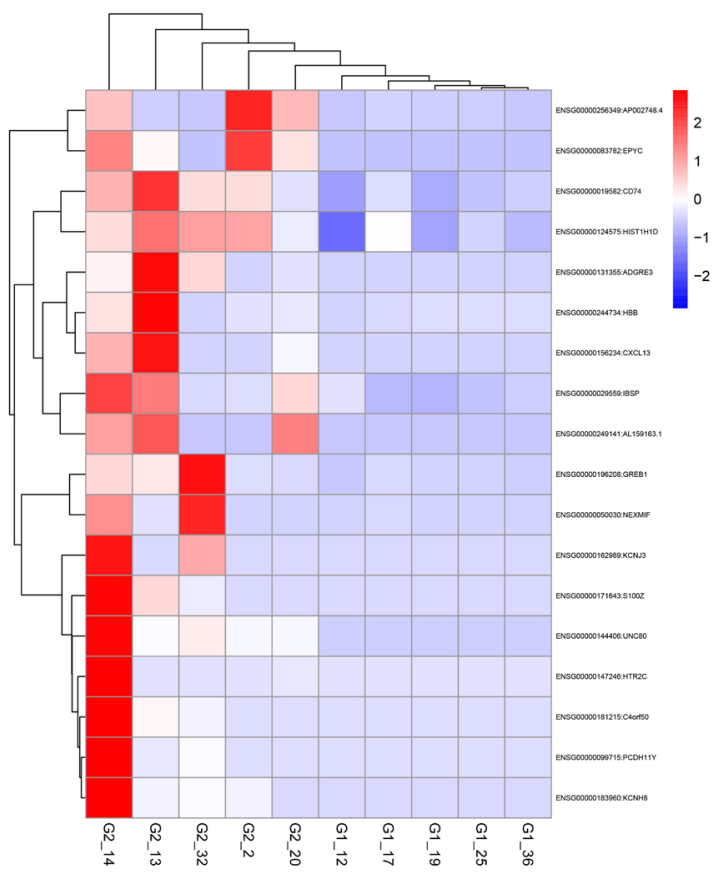

Figure 1 The profile of differentially expressed genes (DEGs) in osteoblasts (OBs) from patients with multiple myeloma (MM). Volcano plot displays differentially expressed long non-coding RNAs (DElncRNAs) (A) and differentially expressed mRNAs (DEmRNAs) (B). Red and green points correspond to upregulation and downregulation, respectively. Heatmap shows the DElncRNAs (C) and DEmRNAs (D). The color scale represents the expression levels of DElncRNAs/DEmRNAs. Group 2 represents patients with MM, whereas group 1 represents healthy controls.

MM and healthy controls. A total of 69860 lncRNAs and 17868 protein-coding mRNAs were identified. Moreover, 43 lncRNAs (22 upregulated and 21 downregulated) and 18 upregulated mRNA were identified as differentially expressed RNAs in patients with MM (online supplemental tables S2, S3). The Volcano Plot shows important differentially expressed lncRNAs (figure 1A) and mRNAs (figure 1B) between MM and healthy controls. Hierarchical clustering classified lncRNAs (figure 1C) and mRNA (figure 1D) and displayed DEGs between patients with MM and healthy controls according to a similar degree of expression profile.
These findings provided beneficial insights into the pathophysiological mechanisms underlying OB activity suppression in MM.

\section{GO and KEGG analyses}

GO enrichment analysis indicated that the upregulated DEGs were enriched in immune system process (BP), membrane (CC), and protein binding (MF) (figure 2A), whereas the downregulated DEGs were found to be mostly enriched in hormone metabolic process (BP), membrane 
(A) Enriched GO Terms
(Group2_vs_Group1mRNA_up_GO)
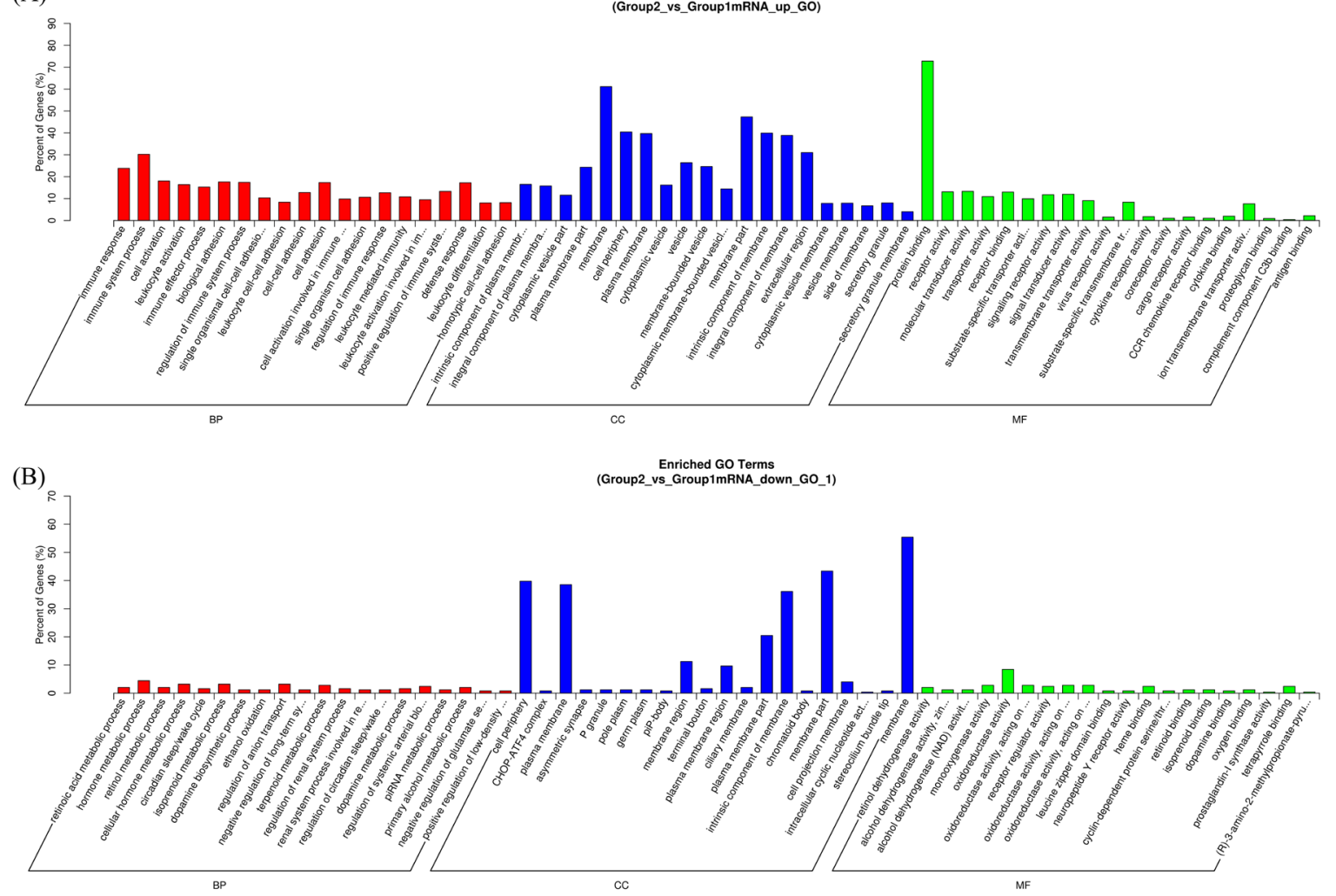

(C)

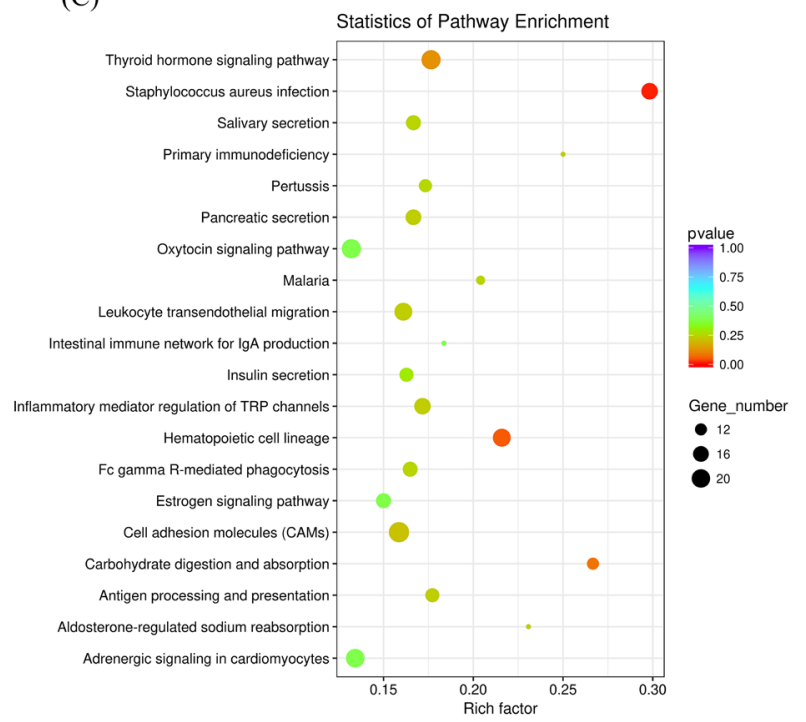

(D)

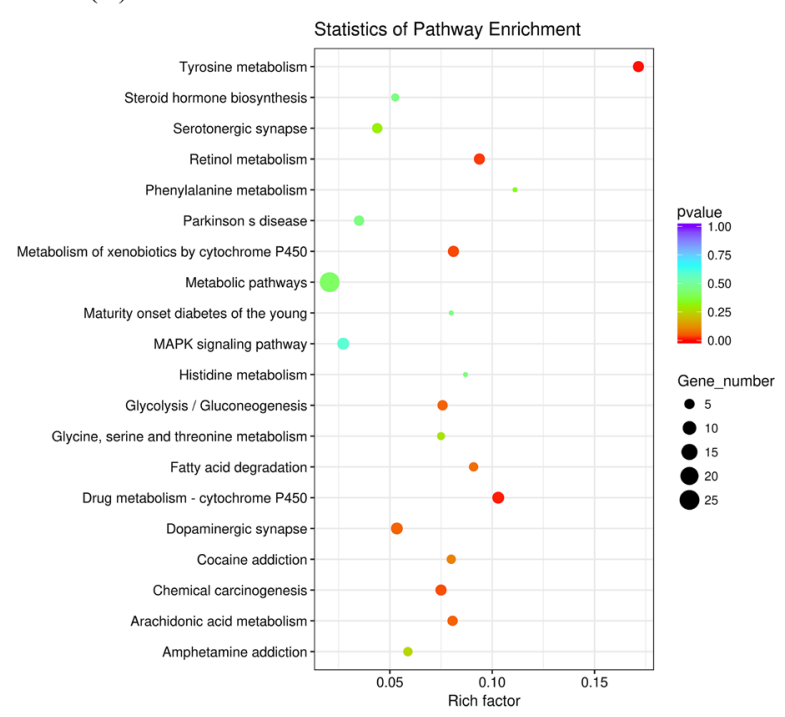

Figure 2 Enrichment analysis of differentially expressed genes (DEGs). Gene Ontology (GO) analysis identifies enriched terms for the upregulated (A) and downregulated DEGs (B). Kyoto Encyclopedia of Genes and Genomes pathways analysis identifies enriched pathways for the upregulated (C) and downregulated genes (D). Group 2 represents patients with multiple myeloma (MM), whereas group 1 represents healthy controls.

(CC), and oxidoreductase activity (MF) (figure 2B). Upregulated DEGs were primarily related to Staphylococcus aureus infection (figure 2C) in the KEGG pathway. The downregulated DEGs were associated with tyrosine metabolism, drug metabolism-cytochrome P450, retinol metabolism, metabolism of xenobiotics by cytochrome P450, and chemical carcinogenesis (figure 2D). 
Table 2 Association analysis of candidate IncRNAs and mRNAs

\begin{tabular}{llll}
\hline IncRNA & Coding gene & Correlation coefficient & P value \\
\hline LINC01473 & CD74 & 0.961098 & $9.56 \mathrm{E}-06$ \\
CMPK2 & CD74 & 0.964356 & $6.76 \mathrm{E}-06$ \\
CSNK1D & HIST1H1D & 0.955178 & $1.67 \mathrm{E}-05$ \\
SMUG1 & HIST1H1D & 0.964098 & $6.96 \mathrm{E}-06$ \\
\hline
\end{tabular}

Correlation coefficient $r>0.95 ; p<0.05$ was considered statistically significant. CMPK2, cytidine/uridine monophosphate kinase 2; CSNK1D, casein kinase 1 delta; HIST1H1D, histone cluster 1 H1 family member d; IncRNA, long non-coding RNA; mRNA, messenger RNA; SMUG1, single-strand-selective monofunctional uracil-DNA glycosylase 1 .

\section{Validation of the sequencing data using quantitative real-time PCR}

We selected several lncRNAs and mRNAs with a high fragment per kilobase million value and a high frequency of the gene occurrence in the test samples as candidates. Four upregulated lncRNAs, namely, LINC01473, cytidine/uridine monophosphate kinase 2 (CMPK2), single-strand-selective monofunctional uracil-DNA glycosylase 1 (SMUG1), and casein kinase 1 delta (CSNK1D) and two upregulated co-expression mRNAs, CD74 and histone cluster $1 \mathrm{H} 1$ family member d (HIST1H1D) were further studied in the following experiments (table 2). The co-expressed lncRNA
mRNA pairs were defined using Pearson's correlation coefficient $r>0.95$ and a $p$ value $<0.05$.

We measured the altered expression of IncRNAs and mRNAs in 18 OBs from BMMNCs of patients with MM to validate the differential expressions of candidate lncRNAs and mRNAs. Levels of LINC01473 (figure 3A) and mRNA CD74 (figure $3 \mathrm{E}$ ) were significantly upregulated in $\mathrm{MM}$ compared with those in healthy controls $(\mathrm{p}=0.047$ and $p=0.016$, respectively). The expression levels of CMPK2, SMUG1, CSNK1D, and HIST1H1D between the two groups were not significantly different (figure 3B, C, D and F).

In addition, the expression of LINC01473 was negatively correlated with serum interleukin-2 (figure $3 \mathrm{G}$ ) and tumor necrosis factor $\alpha$ levels (figure $3 \mathrm{H})(\mathrm{r}=-0.8277, \mathrm{p}<0.001$; $\mathrm{r}=-0.8853, \mathrm{p}<0.001$, respectively). However, the relative expression of mRNA CD74 was positively associated with serum lactic dehydrogenase level in patients with $\mathrm{MM}$ $(r=0.7782, p=0.001)$ (figure 3I).

\section{DISCUSSION}

Bone disease is a major characteristic of $\mathrm{MM}$, and the complication of skeletal-related events affects the quality of life of patient with $\mathrm{MM}$, increases disability and mortality, and results in lower overall survival of the patient.
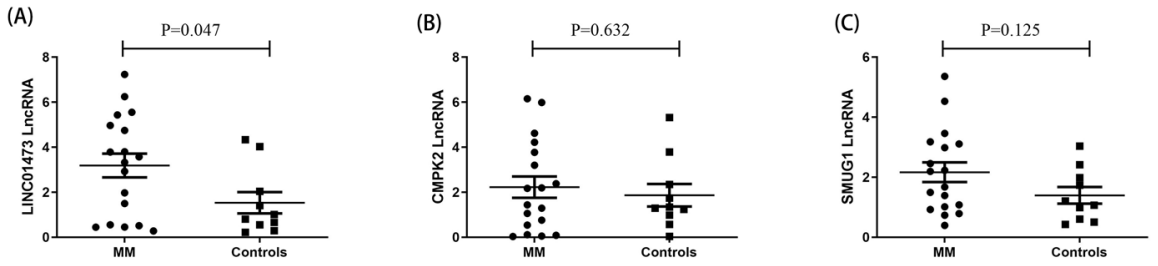
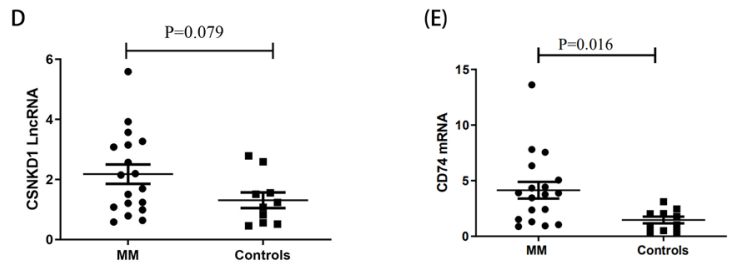

(H)

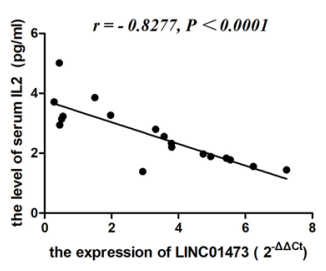

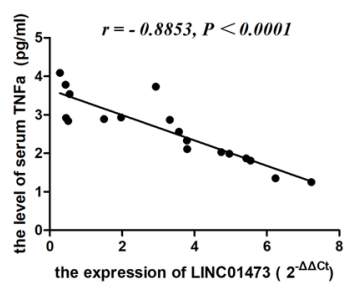

(F)

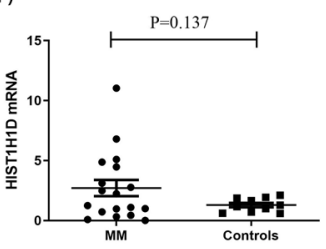

(I)

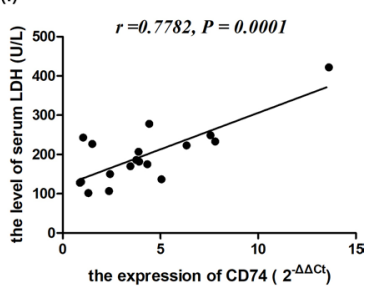

Figure 3 Expression of candidate long non-coding RNAs (IncRNAs) and messenger RNAs (mRNAs) in osteoblasts (OBs) from patients with multiple myeloma (MM) and healthy controls. Expression of LINC01473 in patients with MM is higher than that in healthy controls (A). Levels of cytidine/uridine monophosphate kinase 2 (CMPK2) (B), single-strand-selective monofunctional uracil-DNA glycosylase 1 (SMUG1) (C), and casein kinase 1 delta (CSNK1D) (D) demonstrate no significant changes. Level of CD74 mRNA in patients with MM is higher than that in healthy controls (E). Histone cluster $1 \mathrm{H} 1$ family member d (HIST1H1D) levels demonstrate no significant changes (F). Negative correlation between LINC01473 expression and serum interleukin-2 (IL-2) (G) and tumor necrosis factor $\alpha$ (TNF $\alpha$ ) levels (H). Positive association between mRNA CD74 and serum lactic dehydrogenase (LDH) in patients with MM (I). 
Additionally, abnormal bone remodeling contributes to myeloma bone damage, and suppressed osteoblastogenesis is important for the progression of MM bone disease. Little is known regarding the roles of lncRNAs derived from OBs of patients with MM, which are involved in the occurrence and progression of MM bone disease. In our experiments, we demonstrated that the expression of LINC01473 and its co-expressed mRNA, CD74, in OBs from patients with MM was abnormally elevated. Thus, LINC01473 and CD74 could be involved in MM bone disease and progression of MM.

As a novel lncRNA, the role of LINC01473 remains relatively unknown. CD74 (major histocompatibility complex (MHC) II associated unchanging chain) is a type II transmembrane glycoprotein that plays a critical role in antigen presentation as a chaperone of MHC class II proteins. ${ }^{27}$ CD74 can be detected in traditional antigen-presenting cells (APCs; B cells, dendritic cells and macrophages) and non-traditional APCs (epithelial cells). ${ }^{28}$ CD74 can also act as a receptor for macrophage migration inhibitory factor (MIF) ${ }^{29}$ Many malignant tumors cells expressed upregulated MIF, and its expression level is related to disease evolution. ${ }^{30}$ MIF performs its biological functions through CD74, CXCR2, CXCR4, and CXCR7 receptors in an autocrine and/or paracrine manner. ${ }^{31} 32$ After CD74 binds to MIF, CD44 or CXCR receptors are recruited to induce signal transduction. ${ }^{33}$ Various complexes such as CD74/CD44, CD74/CXCR2, CD74/CXCR4, and CD74/CXCR4/CXCR7 are formed in these receptors. ${ }^{29}$ After cross-linking with CD74, MIF stabilizes $\beta$-catenin, which is a pivotal mediator of the Wnt signaling pathway for $\mathrm{OB}$ differentiation, through the ERK signaling pathway. ${ }^{33}$ The primary OBs demonstrate increased mineralization and calcium deposition. ${ }^{33}$ The researchers found 16 upregulated genes involved in osteogenesis in the gene expression profile of primary OBs. ${ }^{33}$ MIF can also directly inhibit osteoclastogenesis in vitro by activating the tyrosine kinase Lyn. ${ }^{35} 36$ Studies have indicated that CD74-deficient mice exhibit enhanced osteoclastogenesis and bone loss. ${ }^{36}$ In our study, $87 \%$ of patients with MM (20 out of 23 ) experienced bone disease. We observed that the expression of mRNA CD74 in the OBs of patients with MM was greater than that of healthy controls. Thus, elevated levels of CD74 may cause insufficient integration with MIF to accelerate osteoblastogenesis and bone formation in MM.

As a signaling molecule, CD74 is involved in the proliferation, maturation, and anti-apoptosis functions of malignant B cells. ${ }^{37}$ In the development of low-grade to high-grade colon tumors, expression of the MHC II invariant chain displays a significant increase. This indicated that the invariant chain with increased expression demonstrated lower tumor immunogenicity and promoted the occurrence of tumor immunological escape. ${ }^{38}$ Burton et al identified that CD74 was highly expressed in primary malignant myeloma cells (19 out of 22 patients) and myeloma cell lines at the mRNA and protein levels. ${ }^{39}$ However, only 1 out of 19 patients showed HLA-DR positivity. Conversely, HLA-DR and CD74 were co-expressed in most healthy and tumor cells. Abnormal antigen expression on MM cells may lead to tumor immune evasion. Abrahams et al verified that
CD74 mRNA and protein expression was abundant in the plasma cells of patients with MM and the anti-CD74 antibody drug conjugate decrease MM tumor burden. ${ }^{40}$

In conclusion, the results implied that the high expression of CD74 in OBs in patients with MM might be associated with the occurrence of immune escape and disease progression and might cause bone disease. Therefore, targeting CD74 using monoclonal antibody or antibody drug conjugate could reduce MM tumor cell load, and OBs with decreased CD74 expression would recover their osteogenesis ability, thus alleviating MM bone disease and minimizing immune escape. It is also necessary to further discover and verify the roles of LINC01473 and its associated co-expression mRNA, CD74, in osteogenesis in patients with MM. This preliminary work has identified promising targets for alleviating osteogenesis inhibition and accommodating immunological function to prevent and even treat MM bone disease.

Contributors RF designed the project and proofread the manuscript. FP and SY carried out the experiments, analyzed the data and finished the manuscript. $\mathrm{HL}$ and ZL participated in the experimental work. FJ and PC collected clinical data of patients with MM. All authors checked and approved the final manuscript. Guarantor: RF.

Funding This work was supported by the National Natural Science Foundation of China Youth Project (grant no. 82000129, 81900131), the Tianjin Municipal Natural Science Foundation (grant no.18JCQNJC80400), the Tianjin Education Commission Research Project (grant no. 2018KJ043, 2018KJ045), and the Tianjin Science and Technology Planning Project (grant no. 20YFZCSY00060).

Competing interests None declared.

Patient consent for publication Not applicable.

Ethics approval This study was approved by Ethics Committee of Tianjin Medical University General Hospital (ID No. IRB2021-WZ-144). All participants provided written informed consent.

Provenance and peer review Not commissioned; internally peer reviewed. Data availability statement Data are available in a public, open access repository.

Supplemental material This content has been supplied by the author(s). It has not been vetted by BMJ Publishing Group Limited (BMJ) and may not have been peer-reviewed. Any opinions or recommendations discussed are solely those of the author(s) and are not endorsed by BMJ. BMJ disclaims all liability and responsibility arising from any reliance placed on the content. Where the content includes any translated material, BMJ does not warrant the accuracy and reliability of the translations (including but not limited to local regulations, clinical guidelines, terminology, drug names and drug dosages), and is not responsible for any error and/or omissions arising from translation and adaptation or otherwise.

Open access This is an open access article distributed in accordance with the Creative Commons Attribution Non Commercial (CC BY-NC 4.0) license, which permits others to distribute, remix, adapt, build upon this work noncommercially, and license their derivative works on different terms, provided the original work is properly cited, an indication of whether changes were made, and the use is non-commercial. See: http://creativecommons.org/ licenses/by-nc/4.0/.

\section{ORCID iDs}

Fengping Peng http://orcid.org/0000-0002-1761-1399

Zhaoyun Liu http://orcid.org/0000-0002-2730-1562

\section{REFERENCES}

1 Kyle RA, Rajkumar SV. Criteria for diagnosis, staging, risk stratification and response assessment of multiple myeloma. Leukemia 2009;23:3-9.

2 Kyle RA, Gertz MA, Witzig TE, et al. Review of 1027 patients with newly diagnosed multiple myeloma. Mayo Clin Proc 2003;78:21-33. 
3 Terpos E, Ntanasis-Stathopoulos I, Dimopoulos MA. Myeloma bone disease: from biology findings to treatment approaches. Blood 2019;133:1534-9.

4 Qiang Y-W, Chen Y, Stephens O, et al. Myeloma-derived Dickkopf-1 disrupts Wnt-regulated osteoprotegerin and RANKL production by osteoblasts: a potential mechanism underlying osteolytic bone lesions in multiple myeloma. Blood 2008;112:196-207.

5 Delgado-Calle J, Anderson J, Cregor MD, et al. Genetic deletion of SOST or pharmacological inhibition of sclerostin prevent multiple myeloma-induced bone disease without affecting tumor growth. Leukemia 2017;31:2686-94.

6 Takeuchi K, Abe M, Hiasa M, et al. Tgf-Beta inhibition restores terminal osteoblast differentiation to suppress myeloma growth. PLoS One 2010;5:e9870

7 Terpos E, Ntanasis-Stathopoulos I, Christoulas D, et al. Semaphorin 4D correlates with increased bone resorption, hypercalcemia, and disease stage in newly diagnosed patients with multiple myeloma. Blood Cancer J 2018;8:42.

8 Vallet $\mathrm{S}$, Mukherjee S, Vaghela N, et al. Activin A promotes multiple myelomainduced osteolysis and is a promising target for myeloma bone disease. Proc Natl Acad Sci U SA 2010;107:5124-9.

9 Huynh NPT, Anderson BA, Guilak F, et al. Emerging roles for long noncoding RNAs in skeletal biology and disease. Connect Tissue Res 2017:58:116-41.

10 Johnsson P, Lipovich L, Grandér D, et al. Evolutionary conservation of long non-coding RNAs; sequence, structure, function. Biochim Biophys Acta 2014;1840:1063-71

11 Yang N, Chen J, Zhang $\mathrm{H}$, et al. LncRNA OIP5-AS1 loss-induced microRNA-410 accumulation regulates cell proliferation and apoptosis by targeting KLF10 via activating PTEN/PI3K/Akt pathway in multiple myeloma. Cell Death Dis 2017:8:e2975

12 Cho S-F, Chang YC, Chang C-S, et al. MALAT1 long non-coding RNA is overexpressed in multiple myeloma and may serve as a marker to predict disease progression. BMC Cancer 2014;14:809.

13 Meng Y-B, He X, Huang Y-F, et al. Long noncoding RNA CRNDE promotes multiple myeloma cell growth by suppressing miR-451. Oncol Res 2017:25:1207-14.

14 Samur MK, Minvielle S, Gulla A, et al. Long intergenic non-coding RNAs have an independent impact on survival in multiple myeloma. Leukemia 2018;32:2626-35.

15 Li L, Liu B, Wapinski OL, et al. Targeted disruption of HOTAIR leads to homeotic transformation and gene derepression. Cell Rep 2013;5:3-12.

16 Liang W-C, Fu W-M, Wang Y-B, et al. H19 activates Wnt signaling and promotes osteoblast differentiation by functioning as a competing endogenous RNA. Sci Rep 2016;6:20121.

17 Huang $Y$, Zheng $Y$, Jia L, et al. Long noncoding RNA H19 promotes osteoblast differentiation via TGF- $\beta 1 / \mathrm{Smad3/HDAC}$ signaling pathway by deriving miR675. Stem Cells 2015;33:3481-92.

18 Zhu L, Xu P-C, . Downregulated LncRNA-ANCR promotes osteoblast differentiation by targeting EZH2 and regulating Runx2 expression. Biochem Biophys Res Commun 2013:432:612-7.

19 Bäckesjö C-M, Li Y, Lindgren U, et al. Activation of SIRT1 decreases adipocyte formation during osteoblast differentiation of mesenchymal stem cells. J Bone Miner Res 2006;21:993-1002.

20 Cohen-Kfir $\mathrm{E}$, Artsi $\mathrm{H}$, Levin A, et al. Sirt1 is a regulator of bone mass and a repressor of SOST encoding for sclerostin, a bone formation inhibitor. Endocrinology 2011;152:4514-24.
21 Zhuang W, Ge X, Yang S, et al. Upregulation of IncRNA MEG3 promotes osteogenic differentiation of mesenchymal stem cells from multiple myeloma patients by targeting BMP4 transcription. Stem Cells 2015;33:1985-97.

22 Chen L, Song Z, Huang S, et al. IncRNA DANCR suppresses odontoblast-like differentiation of human dental pulp cells by inhibiting Wnt/ $\beta$-catenin pathway. Cell Tissue Res 2016:364:309-18.

23 Kumar SK, Callander NS, Hillengass J, et al. NCCN guidelines insights: multiple myeloma, version 1.2020. J Nat/ Compr Canc Ne 2019;17:1154-65.

24 Fu R, Gao S, Peng F, et al. Relationship between abnormal osteoblasts and cellular immunity in multiple myeloma. Cancer Cell Int 2014;14:62.

25 Liu H, Peng F, Liu Z, et al. Cyr61/Ccn1 stimulates proliferation and differentiation of osteoblasts in vitro and contributes to bone remodeling in vivo in myeloma bone disease. Int J Oncol 2017;50:631-9.

26 Peng F. Data from: high-throughput sequencing of osteoblast from MM patients and health controls. sequence read Archive, 2021. Available: https:// www.ncbi.nlm.nih.gov/sra/PRJNA760661

27 Momburg F, Koch N, Möller P, et al. Differential expression of la and laassociated invariant chain in mouse tissues after in vivo treatment with IFNgamma. J Immunol 1986;136:940-8.

28 Jankauskas SS, Wong DWL, Bucala R, et al. Evolving complexity of MIF signaling. Cell Signal 2019;57:76-88.

29 Bernhagen J, Calandra T, Mitchell RA, et al. MIF is a pituitary-derived cytokine that potentiates lethal endotoxaemia. Nature 1993;365:756-9.

30 Wadgaonkar R, Somnay K, Garcia JGN. Thrombin induced secretion of macrophage migration inhibitory factor (MIF) and its effect on nuclear signaling in endothelium. J Cell Biochem 2008;105:1279-88.

31 Xie J, Yang L, Tian L, et al. Macrophage migration inhibitor factor upregulates MCP-1 expression in an autocrine manner in hepatocytes during acute mouse liver injury. Sci Rep 2016;6:27665.

32 Shi $X$, Leng $L$, Wang T, et al. CD44 is the signaling component of the macrophage migration inhibitory factor-CD74 receptor complex. Immunity 2006:25:595-606

33 Ranganathan V, Ciccia F, Zeng F, et al. Macrophage migration inhibitory factor induces inflammation and predicts spinal progression in ankylosing spondylitis. Arthritis Rheumatol 2017;69:1796-806.

34 Jacquin C, Koczon-Jaremko B, Aguila HL, et al. Macrophage migration inhibitory factor inhibits osteoclastogenesis. Bone 2009;45:640-9.

35 Mun SH, Oh D, Lee S-K. Macrophage migration inhibitory factor downregulates the RANKL-RANK signaling pathway by activating Lyn tyrosine kinase in mouse models. Arthritis Rheumatol 2014:66:2482-93.

36 Mun SH, Won HY, Hernandez P, et al. Deletion of CD74, a putative MIF receptor, in mice enhances osteoclastogenesis and decreases bone mass. $J$ Bone Miner Res 2013;28:948-59.

37 Stein R, Mattes MJ, Cardillo TM, et al. CD74: a new candidate target for the immunotherapy of B-cell neoplasms. Clin Cancer Res 2007:13:5556s-63.

38 Jiang Z, Xu M, Savas L, et al. Invariant chain expression in colon neoplasms. Virchows Arch 1999:435:32-6.

39 Burton JD, Ely S, Reddy PK, et al. CD74 is expressed by multiple myeloma and is a promising target for therapy. Clin Cancer Res 2004; 10:6606-11.

40 Abrahams CL, Li X, Embry M, et al. Targeting CD74 in multiple myeloma with the novel, site-specific antibody-drug conjugate STRO-001. Oncotarget 2018:9:37700-14 